\title{
WILEY-VCH
}

DOI: 10.1002/ ((please add manuscript number))

Article type: Full Paper

\section{Organic Flexible Memristor with Reduced Operating Voltage and High Stability by Interfacial Control of Conductive Filament Growth}

Sin-Hyung Lee, Hea-Lim Park, Chang-Min Keum, In-Ho Lee, Min-Hoi Kim, and Sin-Doo Lee*

S.-H. Lee, Dr. H.-L. Park, Dr. I.-H. Lee, Prof. S.-D. Lee

School of Electrical Engineering, Seoul National University, 1 Gwanak-ro, Gwanak-ku, Seoul 08826, Republic of Korea

E-mail: 1clab2@snu.ac.kr

Dr. C.-M. Keum

Organic Semiconductor Centre, SUPA, School of Physics and Astronomy, University of St

Andrews, St Andrews, KY16 9SS, United Kingdom

Prof. M.-H. Kim

Department of Creative Convergence Engineering, Hanbat National University, Yuseong-ku, Daejeon 305-719, Republic of Korea

Keywords: organic memristor, polymer electrolyte, electrochemical metallization, conductive filament growth, ion migration, resistive switching

We present the underlying mechanisms for the growth of conductive filaments (CFs) at a metal-polymer electrolyte interface through ion migration in organic electrochemical metallization memristor. We observe that the free volume of voids (nanopores) in the polymer electrolyte serves as the pathways of metal-cations whereas the interfacial topography between an active electrode and a polymer electrolyte determines the nucleation sites of the CFs. The growth kinetics of the CFs and the resultant resistive memory are found to vary with the molecular weight of the polymer electrolyte and the metal protrusions at the interface. Our direct observations show that the free volume of voids of the polymer electrolyte, varied with the molecular weight, dictates the ion transport for the growth and the disruption of the CFs. Our organic ECM-based memristor with a hetero-electrolyte exhibits the high mechanical flexibility, the low switching voltages reduced by about three times compared to those of conventional devices, and the stable memory retention for longer than $10^{4} \mathrm{~s}$ under repeated cycles of bending. 


\section{WILEY-VCH}

\section{Introduction}

Memristors, based on the resistive switching, have been demonstrated as an artificial synapse for neuromorphic computing ${ }^{[1,2]}$. Particularly, organic memristors are promising for flexible neuromorphic electronics due to the mechanical flexibility, biocompatibility, and simple fabrication processes ${ }^{[3,4]}$. It is generally known that the resistive switching can be realized by one of three different origins, i.e. thermochemical process ${ }^{[5,6]}$, valence change ${ }^{[7,8]}$, and electrochemical metallization $(\mathrm{ECM})^{[9-11]}$. Among them, the ECM (called conductive bridging random access memory) has attracted much attention for use in high-density neuromorphic systems due to a high level of the scalability as well as the excellent electrical performance ${ }^{[11-}$ ${ }^{14]}$. Typically, the ECM cells are constructed in a simple metal-insulator-metal configuration where a solid electrolyte is sandwiched between an active metal (e.g. silver or copper) electrode and an inert metal electrode. In such configuration, the resistive switching process is achieved by the growth of conductive filaments (CFs), interconnecting the top and bottom electrodes, through the electrochemical dissolution and accumulation of the atoms of the active metal. In other words, the CF growth determines whether the electric charges can flow through the electrolyte medium between two metal electrodes or not ${ }^{[15,16]}$. Therefore, for the organic-based ECM, it is a central issue to clarify the underlying mechanisms for the CF growth and the resultant resistive switching at the metal-polymer electrolyte interface. In general, the CFs in the ECM are grown at two successive stages through the ion migration; i) the nucleation of the CFs for guiding the ion transport and ii) the subsequent transport of the metal-cations along the pathways across the electrolyte medium ${ }^{[15,16]}$. For the inorganic ECM, several studies have revealed the effect of each stage of the CF growth on the improvement of the resistive switching characteristics ${ }^{[16-18]}$. However, for the case of the organic ECM, the ion migration process, the growth of the CFs, and the behavior of the resultant resistive switching have not been fully explored so far. Note that the CF growth into the free volume of voids (or 


\section{WILEY-VCH}

nanopores) of the polymer electrolyte, formed in a bicontinuous fashion ${ }^{[19,20]}$, is fundamentally different from that into inorganic solid electrolytes (for example, amorphous and crystalline oxides) where the ion transport occurs mainly along grain boundaries or disclinations. Another difference is that the organic ECM memristor requires a relatively high operating voltage inherent to a thick layer of the polymer electrolyte (typically about several hundreds of nanometers $)^{[21]}$ since a thin polymer layer often results in high leakage current ${ }^{[22]}$ with undesirable defects such as pin-holes ${ }^{[21,23]}$. Thus, the reduction of the operating voltage remains challenging for the organic ECM memristor.

In this work, we report on the interfacial mechanisms for the CF growth and the resultant resistive switching characteristics in the organic ECM from the viewpoints of the nucleation and the transport of metal-cations. Considering that the glass transition ${ }^{[24]}$, ion conductivity ${ }^{[25]}$, and charge trapping ${ }^{[26]}$ depend on the molecular weight $\left(M_{\mathrm{w}}\right)$ of the polymer, the $\mathrm{CF}$ nucleation and the ion transport should be substantially altered by the free volume of the polymer electrolyte. In our study, a physical picture of the pathway and migration of metal cations for the growth of the CFs, governing the resistive memory characteristics, is obtained in two polymers with different $M_{\mathrm{w}}$ 's. In Figure 1, the underlying concept behind the change in the free volume of voids (or nanopores), the ion pathway, and the CF growth with changing $M_{\mathrm{w}}$ is schematically illustrated. Based on the experimental results, we fabricate an organic flexible ECM device consisting of a hetero-electrolyte of two layers with different $M_{\mathrm{w}}$ 's. Our ECM device exhibits the high mechanical flexibility, the low voltage switching, the high current on/off ratio and the stable memory retention under repeated cycles of bending. 


\section{WILEY-VCH}

\section{Results and Discussion}

For understanding the essential features of the CF growth at two successive stages discussed above, we fabricated two vertical-type ECM devices with different $M_{\mathrm{w}}$ 's as shown in Figure 1. They were composed of 540 nm-thick electrolytes of poly(methyl methacrylate) (PMMA) ${ }^{\text {27] }}$ with different $M_{\mathrm{w}}$ 's; (i) $M_{\mathrm{w}}=996,000 \mathrm{~g} / \mathrm{mol}$ (Device 1) and (ii) $M_{\mathrm{w}}=120,000 \mathrm{~g} / \mathrm{mol}$ (Device 2). Both the top and bottom electrodes were made of silver. The top electrode of each device was prepared on the electrolyte at the deposition rate $\left(D_{\mathrm{r}}\right)$ of $5.0 \AA / \mathrm{s}$. Details of the fabrication were described in Experimental Section.

In view of the nucleation and the transport of metal-cations, we analyzed the resistive switching behaviors, deduced from the current-voltage $(I-V)$ characteristics, of two organic ECM devices. Figure 2a shows the $I-V$ curves of the two devices measured in the voltagesweeping mode in a step of $1 \mathrm{~V}$. In both cases, from the initial high resistance state (HRS), the typical unipolar resistive switching was observed in the writing process and the erasing process as well at the same voltage polarity. During the first sweep, the current gradually increased from the initial HRS and abruptly increased at a certain voltage, defined as the writing voltage ( $V_{\text {write }}$ ), owing to the formation of the CFs. This indicates that the device was switched from the HRS to the low resistance state (LRS). In the subsequent sweep, the current became much larger than that in the first sweep at the voltages lower than $V_{\text {write }}$ since the growth kinetics of the CFs in the LRS was stabilized in the first sweep. The LRS was then set back to the HRS upon the application of a voltage in the region of the negative differential resistance (NDR). Note that the CF is ruptured by the thermal fuse effect in the NDR ${ }^{[27]}$. Device 1 showed a relatively wider and more stable window of memory than Device 2 as clearly shown in Figure 2a. The current in the HRS of Device 2 was about one order of magnitude larger than that of Device 1 and the current variations of Device 2 with the repetition of the switching cycle were significantly larger than those of Device 1, as shown in 


\section{WILEY-VCH}

Figure S1. Note that owing to the larger free space in the polymer electrolyte with the lower molecular weight ${ }^{[24-26]}$, the more growth sites and metal clusters were produced at the polymer-electrode interface by the excessive migration of metal-cations. As a result, the intrinsic resistance reduced during repeated switching cycles as observed in Device 2. We now discuss the effect of the free volume of voids (or nanpores) on the resistive switching in terms of two characteristic voltages, $V_{\text {write }}$ for the CF growth and the electroforming voltage ( $\left.V_{\text {form }}\right)$ for the percolation paths. In fact, $V_{\text {form }}$ corresponds to the voltage for the initiation and the stabilization of the resistive memory ${ }^{[27-29]}$. The statistical data of $V_{\text {form }}$ and $V_{\text {write }}$ for the two devices with different $M_{\mathrm{w}}$ 's were shown in Figure $2 \mathrm{~b}$. Total of 24 devices (12 for each $M_{\mathrm{w}}$ ) were measured to determine the values of $V_{\text {form }}$ and $V_{\text {write }}$ during 16 cycles. In Device 1 , the average values of $V_{\text {form }}$ and $V_{\text {write }}$ were $51.5 \mathrm{~V}$ and $24.1 \mathrm{~V}$, respectively, and in Device 2, they were 22.5 V and 13.3 V (reduced down by a factor of two) respectively. Note that the ion migration in polymer electrolyte is known to be promoted by the hopping of ions between neighboring vacant $\operatorname{sites}^{[30,31]}$. As illustrated in Figure 1, the free volume of voids varying with $M_{\mathrm{w}}$ opens the pathways for the ion transport and guides the growth of the CFs so that the resistive switching is facilitated by the ion migration in the organic ECM systems. This is consistent with what were observed in our case, meaning that Device 2 with a larger free volume indeed exhibited lower operation voltages than Device 1.

Let us examine the memory retention in relation to the stability of the CFs in the LRS's of Device 1 and Device 2. Figure 3a shows the values of the current of Device 1 and Device 2 as a function of time under ambient conditions at the reading voltage $\left(V_{\text {read }}\right)$ of $1 \mathrm{~V}$. In Device 1 , the current remained essentially constant in the range of the retention time $\left(5 \times 10^{3} \mathrm{~s}\right)$ we studied whereas in Device 2, it became deteriorated gradually in time. In elucidating the degradation mechanism, the retention tests of each device were performed at $V_{\text {read }}=1 \mathrm{~V}$ at three different values of the constant compliance current (CC) as shown in Figure $3 b$. It should be noted that the magnitude of the CC varies with the LRS conductance of the organic 


\section{WILEY-VCH}

memristor from the value lower than one quantum conductance by the size of the CF during the writing process ${ }^{[32-35]}$. At the $\mathrm{CC}$ values of $10^{-7} \mathrm{~A}$ and $10^{-8} \mathrm{~A}$, the read current in Device 1 was unchanged for $400 \mathrm{~s}$ while at $10^{-9} \mathrm{~A}$, it decreased abruptly below $10^{-10} \mathrm{~A}$ after $200 \mathrm{~s}$. In other words, the retention failure occurred beyond 200 s. In Device 2, except for the CC at 10${ }^{7}$ A for $200 \mathrm{~s}$, the read current was monotonically decreased below $10^{-10} \mathrm{~A}$, being an indicative of the gradual disruption of the CFs. Considering that the lateral diffusion of metal atoms in the CFs deteriorates the LRS retention ${ }^{[36,37]}$, it is physically reasonable that the weak memory retention of Device 2 is ascribed to the relatively large free volume of voids in the electrolyte as illustrated in Figure 1. In fact, the nature of the memory retention and erasure in the memristor is essential for developing artificial synapses and selectors for mitigating sneak current paths in crossbar arrays ${ }^{[2]}$.

For the direct visualization of the geometrical distribution of the CFs in each polymer electrolyte, two lateral-type devices with different $M_{\mathrm{w}}$ 's were additionally fabricated. A wetetchable and inert metal, gold, was selected as an inert electrode for better stability (see Figure $\mathrm{S} 1$ in the Supporting Information for details of the fabrication steps). Figure 3c shows the field emission-scanning electron microscopy (FE-SEM) images of two lateral-type devices comprising high $M_{\mathrm{w}}$ (left) and low $M_{\mathrm{w}}$ (right) polymer electrolytes where the electroformed $\mathrm{CFs}$ were visible at the $\mathrm{CC}$ of $10^{-7} \mathrm{~A}$. After the writing process, several CFs bridging two metal electrodes were observed in the device with high $M_{\mathrm{w}}$ as shown in Figure 3c. In contrast, in the device with low $M_{\mathrm{w}}$, metal nano-islands formed through the lateral diffusion of metal atoms were clearly seen. These features of the kinetics of the CFs lead directly to the characteristics of the memory retention of Device 1 and Device 2 as shown in Figure 3a. Note that besides the roughness at the inert electrode influencing the CF growth, the stability of the $\mathrm{CF}$ is mainly governed by the mechanical characteristics of the CF and the electrolyte ${ }^{[36,37]}$. As another central issue on the organic ECM, we discuss the relative role of the ion migration to the electrochemical redox reaction on the CF growth. This serves as a guideline for the 


\section{WILEY-VCH}

reduction of the operation voltage of the ECM device. If the redox reaction were dominant, the operating voltages, $V_{\text {form }}$ and $V_{\text {write, }}$ should be nonlinear with the electrode spacing according to the Butler-Volmer equation ${ }^{[36,38]}$. In the organic ECM, since the ion conductivity is substantially low and the polymer electrolyte layer is relatively thick, the drift of metalcations is expected to be much slower than that in the inorganic $\mathrm{ECM}^{[16,39]}$. We determined the dependences of $V_{\text {form }}$ and $V_{\text {write }}$ on the electrode spacing in the lateral-type device configuration (see Figure S2 in the Supporting Information for details of the fabrication steps). As shown in Figure $\mathrm{S} 3$, both $V_{\text {form }}$ and $V_{\text {write }}$ were found to linearly depend on the electrode spacing. This implies that in contrast to inorganic ECM devices ${ }^{[17,18]}$, the migration of metalcations rather than the redox reaction predominantly governs the CF growth in the organic ECM. It is consistent with the results in Figure $2 b$.

Regarding the resistive switching of the organic ECM device, the localization of the growth sites of the CFs on the active electrode plays a critical role in the migration of metal-cations from the metal-polymer electrolyte interface within the context of the local electric field enhancement (LEFE). For the LEFE, we introduced an interpenetrating interface between an active metal electrode and a polymer electrolyte by adjusting the $D_{\mathrm{r}}$ of the metal. Two vertical-type organic ECM devices were fabricated using $540 \mathrm{~nm}$-thick PMMA layers with different $M_{\mathrm{w}}$ 's; (i) $M_{\mathrm{w}}=996,000 \mathrm{~g} / \mathrm{mol}$ (Device 3) and (ii) $M_{\mathrm{w}}=120,000 \mathrm{~g} / \mathrm{mol}$ (Device 4). The active electrode of each device was prepared on the electrolyte at the deposition rate of $D_{\mathrm{r}}$ $=0.1 \AA / \mathrm{s}$. Figure $4 \mathrm{a}$ shows the cross-sectional views of the FE-SEM images showing the interface between the top electrode and the polymer electrolyte of the two devices. Note that at a relatively low deposition rate ${ }^{[40,41]}$, protrusions were clearly produced at the interface only in Device 4 (but not in Device 3). At a relatively high $D_{\mathrm{r}}$, no protrusion was produced irrespective of $M_{\mathrm{w}}$ (see Figure S4 in the Supporting Information for Device 1 and Device 2). This is attributed to the fact that the diffusion of metal atoms into the polymer is promoted through the weak metal-polymer interaction in the voids in the polymer ${ }^{[40,41]}$. The higher $M_{\mathrm{w}}$ 


\section{WILEY-VCH}

leads to less interpenetration of metal atoms into the polymer and thus prevents from forming the protrusions regardless of the deposition rate. To investigate the effect of the protrusions on the resistive switching by the LEFE, we measured the $I-V$ characteristics of Device 4 . In the statistical data shown in Figure $4 \mathrm{~b}$, the values of $V_{\text {form }}$ were measured in 12 devices and the values of $V_{\text {write }}$ were measured in one representative device tested for 16 cycles. A typical behavior of the unipolar resistive switching was shown in Figure S5. In the presence of the protrusions for the LEFE, compared to $V_{\text {form }}=22.5 \mathrm{~V}$ and $V_{\text {write }}=13.3 \mathrm{~V}$ for Device 2 in Figure $2 \mathrm{~b}$, both $V_{\text {form }}$ and $V_{\text {write }}$ for Device 4 were significantly decreased to $14.4 \mathrm{~V}$ and $8.5 \mathrm{~V}$, respectively, as shown in Figure $4 \mathrm{~b}$. This is in analogy to the case that metal nanocrystals in a solid electrolyte serve as the CF growth sites $^{[42]}$.

As a prototype device, we fabricated a flexible organic ECM memristor with a heteroelectrolyte for the low voltage operation as shown in Figure 5a. The hetero-electrolyte was composed of an interfacial layer of low $M_{\mathrm{w}}$ polymer for the protrusions and a bulk layer of high $M_{\mathrm{w}}$ polymer for the metal-cation transport. Note that the high $M_{\mathrm{w}}$ polymer prevents the lateral diffusion of the $\mathrm{CF}$ and the low $M_{\mathrm{w}}$ polymer enables to produce the protrusions for the LEFE and the localization of the growth sites for the CFs. The expanded image in Figure 5a represents the cross-sectional view of the FE-SEM image of our device with the heteroelectrolyte. The total thickness (about $540 \mathrm{~nm}$ ) of the electrolyte was the same as that of Device 1. As shown in Figures 5b, well-defined memory characteristics of the distinct binary nature were observed in the unipolar mode. The current ratio from the LRS to the HRS was about $10^{4}$ at $V_{\text {read }}=1 \mathrm{~V}$. This is much higher than that of the Ag/PMMA-based memristor reported previously ${ }^{[22]}$, which is one of typical ECM memristors ${ }^{[43,44]}$. Note that the thickness of the polymer electrolyte predominantly governs the leakage current of the HRS in the organic ECM device. A sufficiently thick polymer electrolyte of our flexible device contributes to the high current on/off ratio with the low conductance of the HRS. The current level of the HRS was stably maintained for 50 switching cycles without degradation. shown in 


\section{WILEY-VCH}

Figure $4 \mathrm{~b}$, the values of $V_{\text {form }}$ were measured in 12 devices and the values of $V_{\text {write }}$ were measured in one representative device tested for 16 cycles. Figure $5 \mathrm{c}$ shows the statistical data of $V_{\text {form }}$ (top) measured in 12 devices and those of $V_{\text {write }}$ (bottom) in one representative device tested for 16 cycles. The values of $V_{\text {form }}=15.4 \mathrm{~V}$ and $V_{\text {write }}=8.7 \mathrm{~V}$ are about one third of those of Device 1, being similar to those of the devices based on inorganic materials ${ }^{[45,46]}$. Moreover, irrespective of the rigid or flexible substrate, the reduction of the operating voltage was indeed achieved by the hetero-electrolyte, being used in our device architecture, as shown in Figure S6.

We further evaluated the electrical reliability (the memory retention) and the mechanical stability (the durability) of our flexible ECM memristor. Figure 6a shows the memory retention of both the HRS and the LRS at $V_{\text {read }}=1 \mathrm{~V}$ in time under ambient conditions. Clearly, no considerable degradation was observed for $10^{4} \mathrm{~s}$ in the range measured in other works $^{[47,48]}$. Figure $6 \mathrm{~b}$ shows the current variations measured at $1 \mathrm{~V}$ after each cycle of the writing and erasing processes for 600 cycles in the voltage scan from 0 to $9 \mathrm{~V}$ and 9 to $15 \mathrm{~V}$. The observed small fluctuations of the current in the two memory states (the HRS and the LHS) are within the range of the inherent offset between two successive measurements. It should be noted that the switching failure from the LRS to the HRS in the ECM device was observed previously in the voltage-sweeping mode rather than the pulse mode ${ }^{[37]}$. Compared to other ECM devices ${ }^{[37,43]}$, our device indeed exhibits the good reliability in the voltagesweeping mode.

The mechanical flexibility of our device, fabricated on the PEN substrate, under bending deformations was shown in Figure 7a. Figure $7 \mathrm{~b}$ shows the memory retention of our device measured under inward and outward bending deformations with the radius of curvature $r_{\mathrm{b}}=1$ $\mathrm{cm}$. During the bending and relaxing processes for three times in each interval of $100 \mathrm{~s}$, the current in the LRS decreased slightly due to possible defects involved in the electrode. During 


\section{WILEY-VCH}

both inward and outward bending deformations, the current levels in the two memory states remained fairly constant in time for $8 \times 10^{2} \mathrm{~s}$.

\section{Conclusion}

We demonstrated an organic flexible ECM memristor with low voltage operation and high stability through the interfacial control of the CF growth. In the organic ECM, the free volume of random voids (nanopores) in the polymer electrolyte serves as not only the ion pathways for the CF growth but also the vacancy deteriorating the CF stability. It was found that the metal-polymer electrolyte interface with protrusions plays a critical role in the localization of the nucleation sites, the LEFE for the kinetics of the CFs, and the resultant resistive switching characteristics. Our organic ECM memristor with a hetero-electrolyte layer, comprising the interfacial layer of the lower $M_{\mathrm{w}}$ polymer and the bulk layer of the higher $M_{\mathrm{w}}$ polymer, showed the stable switching and retention of the resistive memory under repeated cycles of bending. The operating voltages remained essentially unchanged to be about $15.4 \mathrm{~V}$ for $V_{\text {form }}$ and $8.7 \mathrm{~V}$ for $V_{\text {write, }}$ reduced by about three times compared to those of conventional ECM devices. The observed physical mechanisms for the CF growth at the interface between the metal electrode and the polymer electrolyte enable to design and construct a new class of organic-based flexible neuromorphic devices. 


\section{WILEY-VCH}

\section{Experimental Section}

Characterization: The electrical properties of our devices were measured using a semiconductor parameter analyzer (HP4155A, Hewlett-Packard Co.) under the ambient environment. The accuracy of the current level in our electrical measuring system is $10^{-12} \mathrm{~A}$. In all the electrical measurements, the bottom electrode was grounded and the top electrode was used for the application of the scanning voltage. The geometrical profiles of our devices were measured using a surface profiler (alpha step 500, KLA-Tencor). The morphology of the CFs in the device was characterized using the FE-SEM (S-4800, Hitach) Fabrication of devices: For non-flexible memristors, two different vertical-type devices, comprising poly(methyl methacrylate) (PMMA, Sigma-Aldrich Korea) with $M_{\mathrm{w}}=996,000$ $\mathrm{g} / \mathrm{mol}$ and $120,000 \mathrm{~g} / \mathrm{mol}$, were fabricated. For different $M_{\mathrm{w}}$ 's, the two devices were fabricated under different conditions of the deposition rate for the top electrode (one at the higher rate of $5.0 \AA / \mathrm{s}$ and the other at the lower rate of $0.1 \AA / \mathrm{s}$ ). A prototype of an organic flexible memristor was fabricated using a hetero-electrolyte layer composed of PMMA with $M_{\mathrm{w}}=120,000 \mathrm{~g} / \mathrm{mol}$ for the interfacial layer and $996,000 \mathrm{~g} / \mathrm{ml}$ for the bulk layer. Details of the fabrication steps of our devices are as follows. A substrate (glass for non-flexible devices or polyethylene naphthalate (PEN) for a prototype of a flexible device) was cleaned under ultra-sonication in acetone, isopropyl alcohol, methanol, and deionized water in sequence for $10 \mathrm{~min}$ each. A bottom electrode was made of silver $(\mathrm{Ag})$ of $50 \mathrm{~nm}$ thick by thermal deposition over the entire substrate at the rate of $0.5 \AA / \mathrm{s}$ under the pressure of $10^{-6}$ Torr. For polymer electrolyte layers, two different PMMAs, one with $M_{\mathrm{w}}=996,000 \mathrm{~g} / \mathrm{mol}$ and the other with $M_{\mathrm{w}}=120,000 \mathrm{~g} / \mathrm{mol}$, were dissolved in anisole at the concentration of $8 \mathrm{wt} \%$ and 11 $\mathrm{wt} \%$, respectively. The PMMA solution was spin-coated onto the Ag electrode at the spinning rate of $3000 \mathrm{rpm}$ for $60 \mathrm{~s}$. In the case of the flexible device, for the preparation of the bulk layer, the PMMA solution with $M_{\mathrm{w}}=996,000 \mathrm{~g} / \mathrm{mol}$ in anisole at $6 \mathrm{wt} \%$ was spin-coated on the Ag electrode at the rate of $2000 \mathrm{rpm}$ for $60 \mathrm{~s}$. For the interfacial layer, the PMMA solution 


\section{WILEY-VCH}

with $M_{\mathrm{w}}=120,000 \mathrm{~g} / \mathrm{mol}$ in anisole at $5 \mathrm{wt} \%$ was then spin-coated on the top of the bulk layer at the rate of $4000 \mathrm{rpm}$ for $60 \mathrm{~s}$. Note that each PMMA layer was soft-baked at $110{ }^{\circ} \mathrm{C}$ for $60 \mathrm{~min}$ to remove the residual solvent after spin-coating. All the single electrolytes for the non-flexible devices were $540 \mathrm{~nm}$ thick. For the flexible device, the bulk layer and the interfacial layer for the hetero-electrolyte were $420 \mathrm{~nm}$ and $120 \mathrm{~nm}$ thick, respectively. As the top electrode, a $50 \mathrm{~nm}$-thick Ag layer was prepared by thermal evaporated at the rate of $5 \AA / \mathrm{s}$ (some of the non-flexible devices) or $0.1 \AA / \mathrm{s}$ (the remaining non-flexible devices and the flexible device) under the pressure of $10^{-6}$ Torr. The physical size of all devices was $0.5 \mathrm{~mm}$ $\times 0.5 \mathrm{~mm}$.

\section{Supporting Information}

Supporting Information is available from the Wiley Online Library or from the author.

\section{Acknowledgements}

This work was supported in part through the BK21 Program funded by Ministry of Education of Korea

Received: ((will be filled in by the editorial staff))

Revised: ((will be filled in by the editorial staff)) Published online: ((will be filled in by the editorial staff)) 


\section{WILEY-VCH}

References

[1] J. J. Yang, D. B. Strukov, D. R. Stewart, Nat. Nanotechnol. 2013, 8, 13.

[2] Z. Wang, S. Joshi, S. E. Savelev, H. Jiang, R. Midya, P. Lin, M. Hu, N. Ge, J. P.

Strachan, Z. Li, Q. Wu, M. Barnell, G.-L. Li, H. L. Xin, R. S. Williams, Q. Xia, J. J. Yang, Nat. Mater. 2017, 16, 101.

[3] G. Liu, C. Wang, W. Zhang, L. Pan, C. Zhang, X. Yang, F. Fan, Y. Chen, R.-W. Li, Adv. Electron. Mater. 2016, 2, 1500298.

[4] Y. van de Burgt, A. Melianas, S. T. Keene, G. Malliaras, A. Salleo, Nat. Electron. 2018, 1,386 .

[5] D. Ielmini, R. Bruchhaus, R. Waser, Phase Transitions 2011, 84, 570.

[6] Y. Luo, D. Zhao, Y. Zhao, F.-K. Chiang, P. Chen, M. Guo, N. Luo, X. Jiang, P. Miao, Y. Sun, A. Chen, Z. Lin, J. Li, W. Duan, J. Cai, Y. Wang, Nanoscale 2015, 7, 642.

[7] N. Lu, L. Li, P. Sun, M. Wang, Q. Liu, H. Lv, S. Long, M. Liu, J. Phys. D: Appl. Phys. 2015, 48, 065101.

[8] N. Lu, L. Li, P. Sun, M. Wang, Q. Liu, H. Lv, S. Long, W. Banerjee, M. Liu, Mater. Res. Express 2015, 2, 046304.

[9] S. Liu, N. Lu, X. Zhao, H. Xu, W. Banerjee, H. Lv, S. Long, Q. Li, Q. Liu, M. Liu, Adv. Mater. 2016, 28, 10623.

[10] J. Yoo, J. Park, J. Song, S. Lim, H. Hwang, Appl. Phys. Lett. 2017, 111, 063109.

[11] G. Du, Z. Chen, Q. Mao, Z. Ji, Appl. Phys. Lett. 2017, 110, 093507.

[12] P. Sun, N. Lu, L. Li, Y. Li, H. Wang, H. Lv, Q. Liu, S. Long, S. Liu, M. Liu, Sci. Rep. 2015, 5, 13504.

[13] N. Lu, P. Sun, L. Li, Q. Liu, S. Long, H. Lv, M. Liu, Chin. Phys. B 2016, 25(5), 056501.

[14] S. Choi, S. H. Tan, Z. Li, Y. Kim, C. Choi, P.-Y. Chen, H. Yeon, S. Yu, J. Kim, Nat. Mater. 2018, 17, 335. 


\section{WILEY-VCH}

[15] X. Zhao, H. Xu, Z. Wang, Z. Xu, C. Zhang, G. Wang, W. Liu, J. Ma, Y. Liu, J. Mater. Chem. C 2017, 5, 5420.

[16] I. Valov, R. Waser, J. R. Jameson, M. N. Kozicki, Nanotechnology 2011, 22, 254003.

[17] C. Schindler, I. Valov, R. Waser, Phys. Chem. Chem. Phys. 2009, 11, 5974.

[18] C. Schindler, G. Staikov, R. Waser, Appl. Phys. Lett. 2009, 94, 072109.

[19] C. Nagel, E. Schmidtke, K. Gunther-Schade, D. Hofmann, D. Fritsch, T. Strunskus, F. Faupel, Macromolecules 2000, 33, 2242.

[20] P. Singh, R. Kumar, R. Singh, A. Roychowdhury, D. Das, Appl. Surf. Sci. 2015, 328, 482.

[21] B. C. Jang, H. Seong, S. K. Kim, J. Y. Kim, B. J. Koo, J. Choi, S. Y. Yang, S. G. Im, S.-Y. Choi, ACS Appl. Mater. Interfaces 2016, 8, 12951.

[22] J. Mangalam, S. Agarwal, A. N. Resmi, M. Sundararajan, K. B. Jinesh, Org. Electron. 2016, 29, 33.

[23] Y.-Y. Noh, H. Sirringhaus, Org. Electron. 2009, 10, 174.

[24] G. Pezzin, F. Zilio-Grandi, P. Sanmartin, Eur. Polym. J. 1970, 6, 1053.

[25] A. A. Teran, M. H. Tang, S. A. Mullin, B. P. Balsara, Solid State Ionics 2011, 203, 18.

[26] H. H. Choi, W. H. Lee, K. Cho, Adv. Funct. Mater. 2012, 22, 4833.

[27] S.-H. Lee, I.-H. Lee, C.-M. Keum, M.-H. Kim, C. Kim, S.-D. Lee, Org. Electron. 2017, 51,357 .

[28] C.-Y. Liu, J.-J. Huang, C.-H. Lai, C.-H. Lin, Nanoscale Res. Lett. 2013, 8, 156.

[29] H. Sun, Q. Liu, C. Li, S. Long, H. Lv, C. Bi, Z. Huo, L. Li, M. Liu, Adv. Funct. Mater. 2014, 24, 5679 .

[30] M. C. Lonergan, A. Nitzan, M. A. Ratner, D. F. Shriver, J. Chem. Phys. 1995, 103, 3253.

[31] S. Das, A. Ghosh, J. Phys. D: Appl. Phys. 2016, 49, 235601. 


\section{WILEY-VCH}

[32] Y. Wang, Q. Liu, S. Long, W. Wang, Q. Wang, M. Zhang, S. Zhang, Y. Li, Q. Zuo, J. Yang, M. Liu, Nanotechnology 2010, 21, 045202.

[33] C. Chen, Y. C. Yang, F. Zeng, F. Pan, Appl. Phys. Lett. 2010, 97, 083502.

[34] R. M. Mutiso, J. M. Kikkawa, K. I. Winey, Appl. Phys. Lett. 2013, 103, 223302.

[35] X. Tian, S. Yang, M. Zeng, L. Wang, J. Wei, Z. Xu, W. Wang, X. Bai, Adv. Mater. $\mathbf{2 0 1 4}, 26,22$.

[36] C.-P. Hsiung, H.-W. Liao, J.-Y. Gan, T.-B. Wu, J.-C. Hwang, F. Chen, M.-J. Tsai, ACS Nano 2010, 4, 5414.

[37] X. Zhao, S. Liu, J. Niu, L. Liao, Q. Liu, X. Xiao, H. Lv, S. Long, W. Banerjee, W. Li, S. Si, M. Liu, Small 2017, 1603948.

[38] S. Menzel, R. Waser, Nanoscale 2013, 5, 11003.

[39] R. Waser, R. Dittmann, G. Staikov, K. Szot, Adv. Mater. 2009, 21, 2632.

[40] R. Willecke, F. Faupel, Macromolecules 1997, 30, 567.

[41] F. Faupel, R. Willecke, A. Thran, Mater. Sci. Eng., $R$ 1998, R22, 1.

[42] Q. Liu, S. Long, H. Lv, W. Wang, J. Niu, Z. Huo, J. Chen, M. Liu, ACS Nano 2010, 4, 6162.

[43] S. Gao, C. Song, C. Chen, F. Zeng, F. Pan, J. Phys. Chem. C 2012, 116, 17955.

[44] X. Zhao, M. Li, H. Xu, Z. Wang, C. Zhang, W. Liu, J. Ma, Y. Liu, J. Alloys Compd. 2016, 656, 612 .

[45] G.-S. Park, X.-S. Li, D.-C. Kim, R.-J. Jung, M.-J. Lee, S. Seo, Appl. Phys. Lett. 2007, 91,222103

[46] J. Park, H. Jeon, H. Kim, W. Jang, H. Seo, H. Jeon, RSC Adv. 2014, 4, 61064.

[47] F. M. Simanjuntak, P. Singh, S. Chandrasekaran, F. J. Lumbantoruan, C.-C. Yang, C.J. Huang, C.-C. Lin, T.-Y. Tseng, Semicond. Sci. Technol. 2017, 32, 124003.

[48] F. M. Simanjuntak, S. Chandrasekaran, B. Pattanayak, C.-C. Lin, T.-Y. Tseng, Nanotechnology 2017, 28, 38LT02. 


\section{WILEY-VCH}
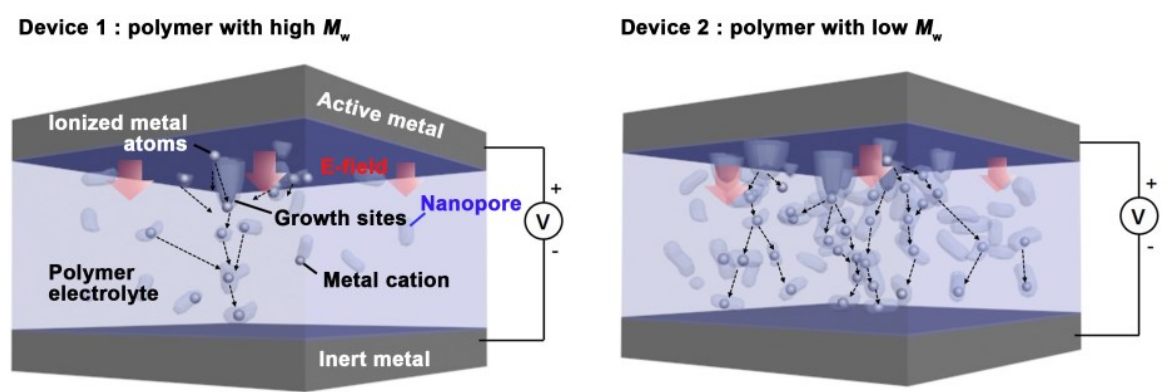

Figure 1. The underlying concept behind the ion migration for the $\mathrm{CF}$ growth into the free volume of voids (or nanopores) in the polymer electrolyte. Two vertical-types of the ECM devices (Device 1 and Device 2) with different $M_{\mathrm{w}}$ 's are illustrated.
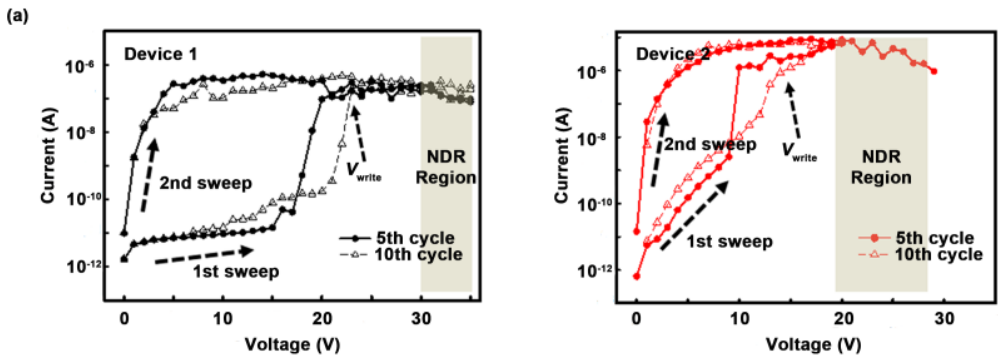

(b)
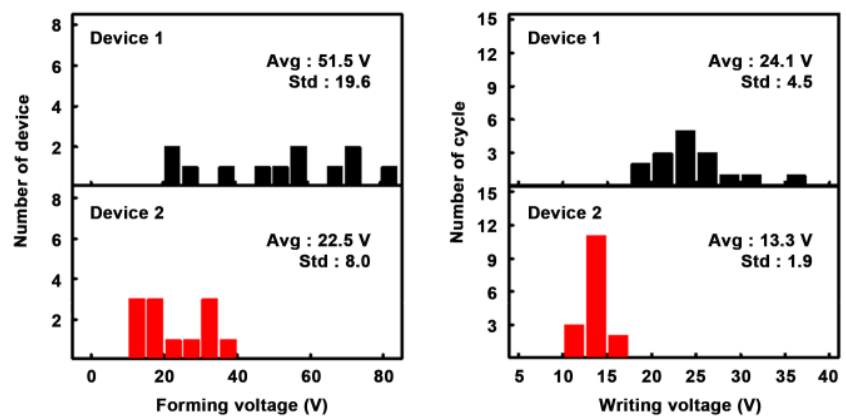

Figure 2. Two vertical-type organic ECM devices with different $M_{\mathrm{w}}$ 's (Device 1 with high $M_{\mathrm{w}}$ and Device 2 with low $M_{\mathrm{w}}$ ) were prepared at the deposition rate of $5.0 \AA / \mathrm{s}$. (a) Currentvoltage $(I-V)$ characteristics of two vertical-type devices (Device 1 with high $M_{\mathrm{w}}$ and Device 2 with low $M_{\mathrm{w}}$ ). The values of $V_{\text {form }}$ of $52 \mathrm{~V}$ in Device 1 and $22 \mathrm{~V}$ in Device 2 for the initiation and the stabilization. (b) Statistical data of the operating voltages ( $V_{\text {form }}$ and $\left.V_{\text {write }}\right)$. Total of 24 devices (12 for each $M_{\mathrm{w}}$ ) were characterized. 


\section{WILEY-VCH}

(a)

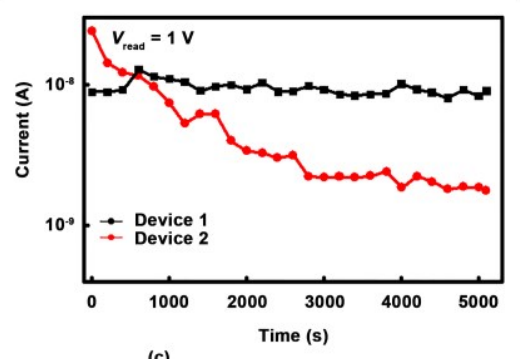

(c)
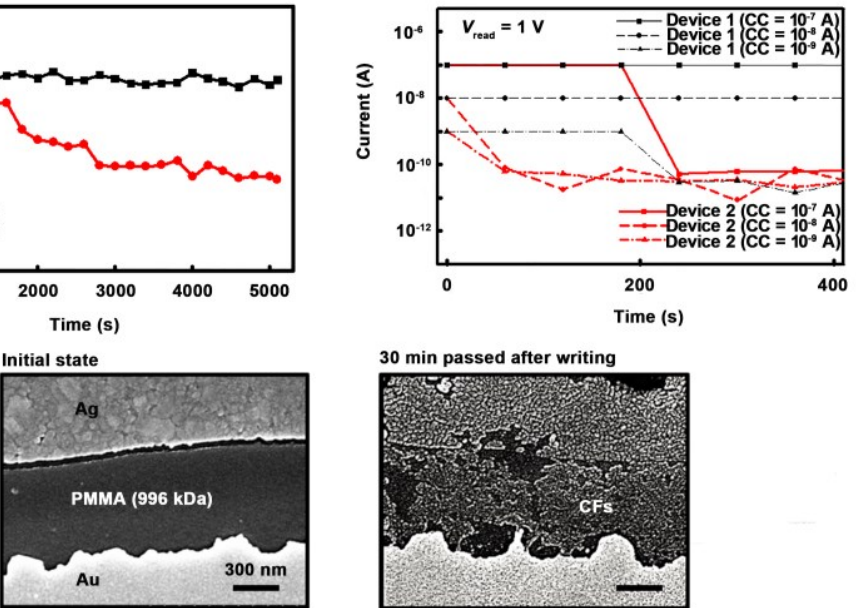

30 min passed after writing

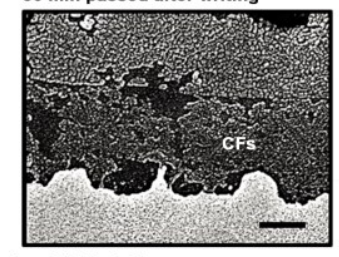

Lateral-type device with high $M$

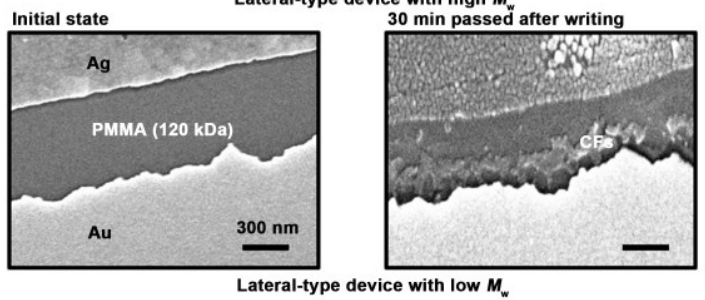

Figure 3. (a) Memory retention characteristics of two vertical-type devices (Device 1 and Device 2) in the LRS at $V_{\text {read }}=1 \mathrm{~V}$. (b) Memory retention tests of Device 1 and Device 2 performed at $V_{\text {read }}=1 \mathrm{~V}$ at three different values of the compliance current (CC). (c) FE-SEM images of two lateral-type devices, one with high $M_{\mathrm{w}}$ (left) and the other with low $M_{\mathrm{w}}$ (right) for the electrolyte, at the $\mathrm{CC}$ of $10^{-7} \mathrm{~A}$ for electroforming the CFs.

(a)
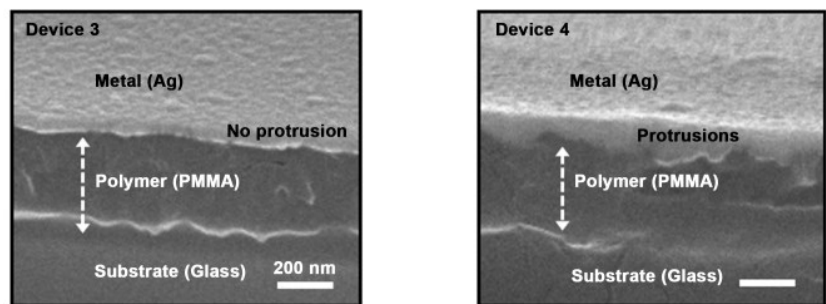

(b)
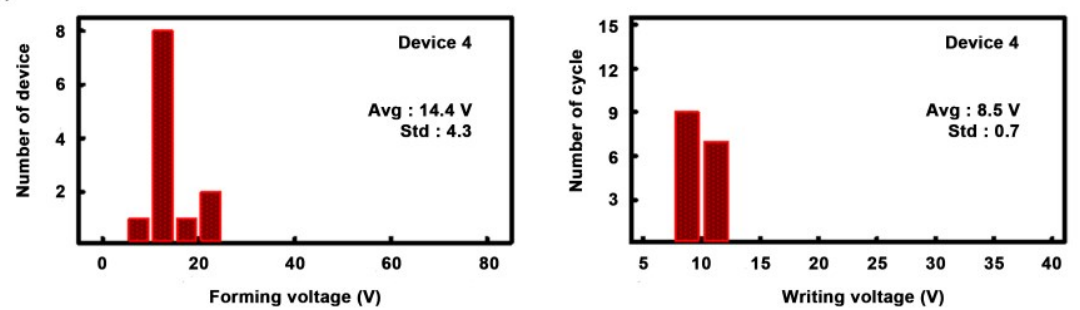

Figure 4. Two vertical-type organic ECM devices with different $M_{\mathrm{w}}$ 's (Device 3 with high $M_{\mathrm{w}}$ and Device 4 with low $M_{\mathrm{w}}$ ) were prepared at the deposition rate of $0.1 \AA / \mathrm{s}$. (a) The crosssectional views of the FE-SEM images showing the interfaces between the electrode and the polymer electrolyte of two vertical-type devices (Device 3 and 4). (b) Statistical data of the operating voltages ( $V_{\text {form }}$ and $V_{\text {write }}$ ) of Device 4. 


\section{WILEY-VCH}

(a)

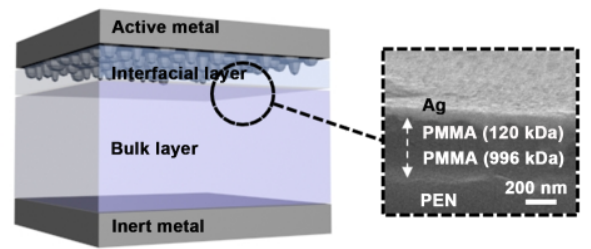

(b)

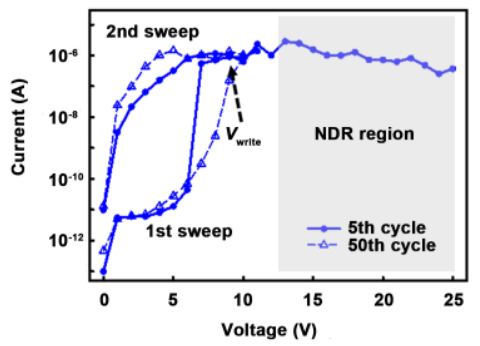

(c)
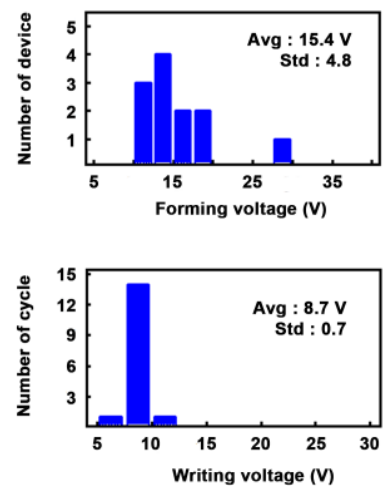

Figure 5. (a) Schematic diagram showing the architecture of our flexible ECM memristor with a hetero-electrolyte consisting of two layers with high $M_{\mathrm{w}}$ and low $M_{\mathrm{w}}$. The expanded image shows the cross sectional view of the FE-SEM image of the hetero-electrolyte. (b) Current-voltage $(I-V)$ characteristics in the voltage-sweeping mode. $V_{\text {form }}=13 \mathrm{~V}$ for the initiation and the stabilization. (c) Statistical data of the operating voltages ( $V_{\text {form }}$ and $V_{\text {write) }}$ ).
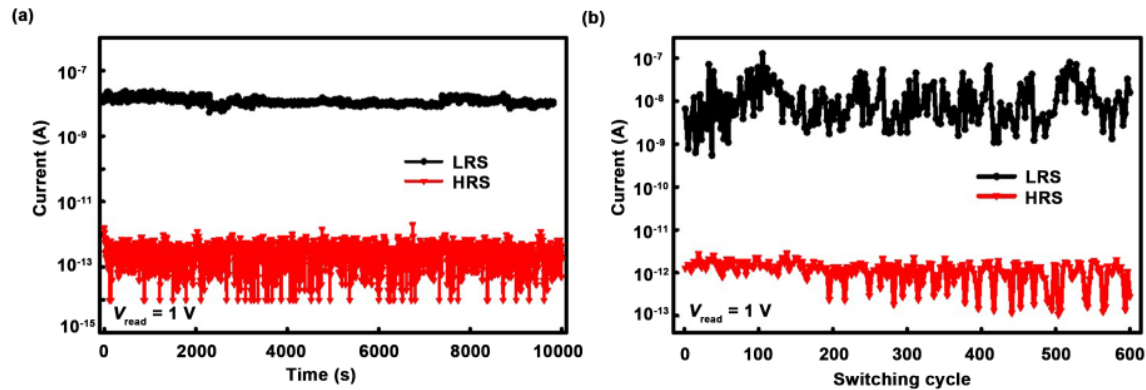

Figure 6. (a) Retention characteristics of our flexible ECM memristor at $V_{\text {read }}=1$ V. (b) Durability characteristics as a function of the switching cycle.
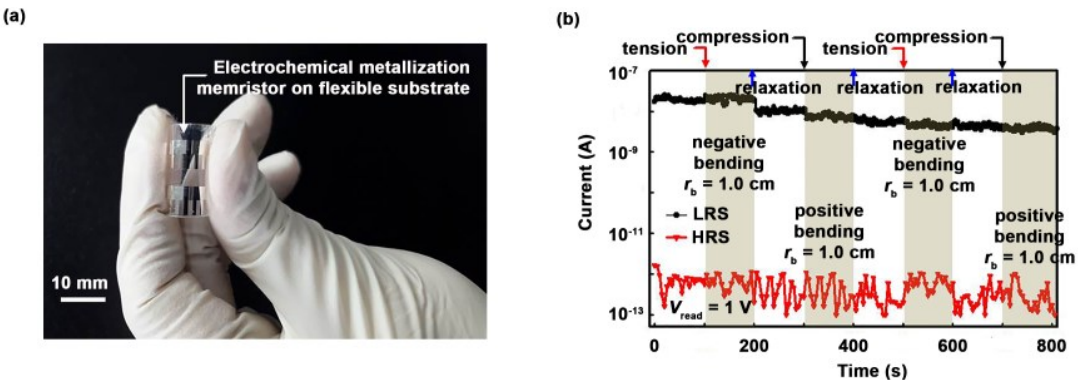

Figure 7. (a) A photograph of our flexible ECM memristor fabricated on the PEN substrate (2 $\mathrm{cm} \times 2 \mathrm{~cm}$ in size) under bending. (b) The read current in time under the positive (inward) and negative (outward) bending deformations with the curvature radius $r_{\mathrm{b}}=1 \mathrm{~cm}$. 